\title{
Morphometric analysis of the visceral yolk sac endoderm in the rat in vivo and in vitro
}

\author{
M. Gupta, A.P. Gulamhusein and F. Beck \\ Department of Anatomy, Leicester University Medical School, University Road, \\ Leicester LE1 7RH, U.K.
}

\begin{abstract}
Summary. Rat embryos together with their visceral yolk sac were grown in vitro during the early period of organogenesis from days 9.5 to 11.5 and the ultrastructural morphology of the visceral yolk sac cells was compared with that in vivo at the beginning and at the end of the culture period. Identical areas of the visceral yolk sac endodermal cells were analysed morphometrically. A measure of the functional activity was obtained by comparison of the volume density and surface density of the vacoular system. At 9.5 and 11.5 days, the values obtained were virtually identical for yolk sacs in vivo and in vitro. At 9.5 days, the volume and surface density of the endocytotic vacuoles in the embryonic endoderm was significantly lower than in the visceral yolk sac endoderm.

It is concluded that the digestive function of the yolk sac is almost certainly identical in culture and in vivo and that the cells of the embryonic endoderm do not take a significant part in embryonic nutrition.
\end{abstract}

\section{Introduction}

Preplacental embryonic nutrition is achieved by the uptake and catabolism of maternal macromolecules which constitute the so-called histiotroph. This process takes place in the extra-embryonic membranes. Histiotroph arises from a variety of sources, but chiefly from the breakdown of proliferated endometrial cells, uterine secretion and extravasated maternal blood (Amoroso, 1952). In eutherian mammals, it is the only form of nutrition available to the embryo before the establishment of a chorio-allantoic placenta which transfers material directly from the maternal to the embryonic circulation across a 'placental barrier'. In the rat the chorio-allantoic placenta begins to function at about 11.5 days of gestation, but histiotrophic nutrition continues alongside this until term. The principal site for the final digestion of rat histiotroph is the visceral layer of the yolk sac epithelium (Beck, Lloyd \& Griffiths, 1967) and this membrane persists as a functional entity until full term.

Rat embryos can now be grown routinely with their visceral yolk sac during much of the period of organogenesis (i.e. from Days 9.5 to 11.5 ) in specially prepared rat serum using the roller-tube culture technique (see New, 1978, for review). The rate and quality of embryonic growth and differentiation in vitro is virtually identical to that in vivo (Cockroft, 1976), but it has not been shown that embryonic nutrition is identical in vivo and in vitro.

In the present studies (part of a joint project with the University of Keele), a detailed morphometric analysis was carried out at 9.5 and 11.5 days of gestation to see whether yolk sac activity is truly identical in culture and in vivo. 


\section{Materials and Methods}

\section{Animals}

Male and female Wistar rats were placed together in the evening and vaginal smears were examined for the presence of spermatozoa on the following morning. Rats showing a positive smear were presumed to have mated within $2 \mathrm{~h}$ of midnight $(24: 00 \mathrm{~h})$ on the previous night, conceptuses thus being 0.5 days old at $12: 00 \mathrm{~h}$ (noon).

\section{Embryos}

In vitro. The uterus was quickly removed from 9-5-day-pregnant rats (early head-fold stage) and aseptically transferred to a dish containing Hank's Balanced Salt Solution (Flow Laboratories Ltd., P.O. Box 17, Irvine, Scotland). It was then carefully opened with forceps to expose pear-shaped masses of decidual tissue from which the embryos and their yolk sacs, with a little adherent trophoblast and the ectoplacental cone, were dissected out in fresh Hank's Solution under a dissecting microscope. The outer layer of the yolk sac (Reichert's membrane with attached trophoblast and parietal endoderm) was opened but the visceral yolk sac, amnion and ectoplacental cone were left intact. Between 10 and 30 min after removal from the mother these specimens were fixed in glutaraldehyde (see below). The conceptuses (egg cylinders) prepared in this fashion constituted the in-vitro series because, in contrast to the rapidly fixed in-vivo embryos, they were prepared in Hank's solution and remained in this fluid for up to 30 min. This procedure was identical to that adopted when embryos were cultured for $48 \mathrm{~h}$. Fixation was carried out at the point when incubation in serum would begin in the 11.5-day explanted specimens.

To obtain $11 \cdot 5$-day specimens, similarly prepared $9 \cdot 5$-day egg cylinders were placed in a sterile glass bottle which contained $1 \mathrm{ml}$ of immediately centrifuged heat-inactivated rat serum per embryo (New, 1978). There were 4-6 egg cylinders in each bottle. The bottles were stoppered and placed horizontally on rollers in an incubator at $37^{\circ} \mathrm{C}$ at $40-50 \mathrm{rev} . / \mathrm{min}$ for $48 \mathrm{~h}$ (New, 1978). For the first $24 \mathrm{~h}$ of the culture period, the embryos were gassed with a gas mixture containing $5 \% \mathrm{O}_{2}-5 \% \mathrm{CO}_{2}-90 \% \quad \mathrm{~N}_{2}$. This was replaced with $20 \% \mathrm{O}_{2}-5 \% \mathrm{CO}_{2}-75 \% \mathrm{~N}_{2}$ between 24 and $36 \mathrm{~h}$ of culture and with $40 \% \mathrm{O}_{2}-5 \% \mathrm{CO}_{2}-55 \% \mathrm{~N}_{2}$ between 36 and $48 \mathrm{~h}$ of culture.

In vivo. Specimens were obtained by killing rats at 9.5 or 11.5 days post coitum. The sterile uterus was immediately transferred to a dish containing heat-inactivated rat serum at $37^{\circ} \mathrm{C}$. In this fluid conceptuses were rapidly dissected out and placed in $3 \%$ glutaraldehyde. Unlike the in-vitro preparations, the in-vivo embryos were never in contact with Hank's solution.

\section{Tissue processing}

All specimens were eventually fixed in $3 \%$ glutaraldehyde in phosphate buffer ( $\mathrm{pH} \mathrm{7.2-7.4)}$ for $3 \mathrm{~h}$ at $4^{\circ} \mathrm{C}$, washed twice in sucrose buffer $(6.82 \mathrm{~g}$ sucrose in $100 \mathrm{ml}$ phosphate buffer, $\mathrm{pH}$ $7 \cdot 2-7.4)$ at a 5 -min interval and left overnight in sucrose buffer at $4^{\circ} \mathrm{C}$. The tissue was then post-fixed in $2 \%$ osmium tetroxide for $1 \mathrm{~h}$ at $4^{\circ} \mathrm{C}$, dehydrated in graded ethanol solutions at room temperature and embedded in araldite.

\section{Tissue orientation}

To compare the endocytotic activity of the yolk sac epithelium in vivo and in vitro, identical areas of the yolk sacs were sectioned at both stages of gestation (Text-fig. 1). 


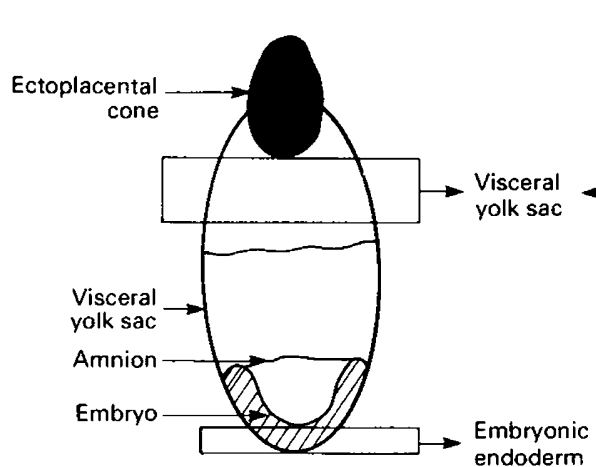

(a)

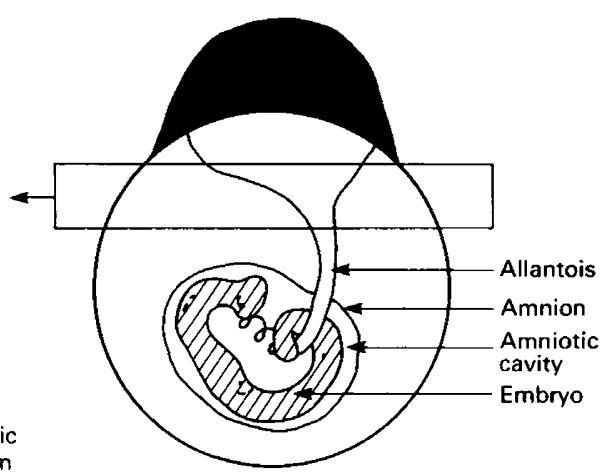

(b)

Text-fig. 1. Diagrams of rat embryos at (a) 9.5 and (b) 11.5 days, showing the area of the visceral yolk sac endoderm and embryonic endoderm studied in vivo and in vitro. Reichert's membrane with attached trophoblast and parietal yolk sac were removed.

\section{Microscopy}

Semithin sections (about $1 \mu \mathrm{m}$ thick) were cut and stained with toluidine blue, and examined under the light microscope to identify the orientation of the yolk sac cells.

Ribbons of ultrathin sections ( $70 \mathrm{~nm}$ thick) were cut from each of the tissue blocks, collected on 200 micromesh copper grids and stained with uranyl acetate and lead citrate. Electron micrographs were taken using a JEOL 100 S electron microscope at an accelerating voltage of 80 $\mathrm{kV}$. Micrographs were taken at 7000 magnification and printed to a final magnification of 21000 .

\section{Stereological analyses}

Volume density $\left(\mathrm{V}_{\mathrm{v}}\right)$ is the volume of a profile of a particular interest expressed as a proportion of its containing volume and is calculated (Williams, 1977) as points over the object profile/total points applied to the micrograph.

In the present studies, measurements were made on photomicrograph prints. The volume density of the endocytotic vacuoles was expressed as a percentage of the total cytoplasmic volume obtained by 'point counting' analysis of electron photomicrographs as developed by Weibel (1969). The intersections of a squared lattice marked on a transparent screen which was superimposed onto each photomicrograph provided the points. The size of the test lattice used was $1 \mathrm{~cm}^{2}$. The procedure was greatly accelerated by using a quantitative analyser (MOP AMO2) and a table top computer (Apple II).

Surface density $\left(S_{v}\right)$ relates the surface area of a cell compartment made up of any specific organelle type to the total cell volume in which it is contained. It is expressed as $S_{v}=2 \cdot I_{L}$ where $\mathbf{L}$ equals the total length of horizontal lines placed in an overlay upon a photomicrograph and $I$ the number of times the lines cut the contours of interest (Williams, 1977). Estimates of membrane area of the endocytotic vacuolar compartment were obtained by counting the number of intersections on the vacuolar membrane. The results are expressed as $\mu \mathrm{m}^{2}$ per $\mu \mathrm{m}^{3}$ of the cytoplasmic volume.

\section{Sampling procedure}

A sample large enough to ensure reasonable levels of accuracy for the measurements of volume density and surface density was obtained by drawing 'Cumulative Mean Plots' 
(Williams, 1977). The sample size required to achieve and remain within $\pm 10 \%$ of the final cumulative mean was taken as the 'Minimal Sample Size' (MSS) for that particular measurement.

Three pregnant rats were used in each experimental group, two conceptuses were taken from each rat and two sections were taken from each conceptus. Photomicrographs of yolk sac endodermal cells were obtained by a random sampling procedure which covered the entire depth of the endodermal cells appearing on the ultrathin sections (Text-fig. 2). Point counting was done on each photomicrograph (see above) and a graph was plotted to show the cumulative means for volume density and surface density. Each of the plots showed that the cumulative mean falls and remains within $10 \%$ of the final value used in our calculations after analysis of 11-14 micrographs (Text-fig. 3). In order to be quite certain of obtaining a representative sample for

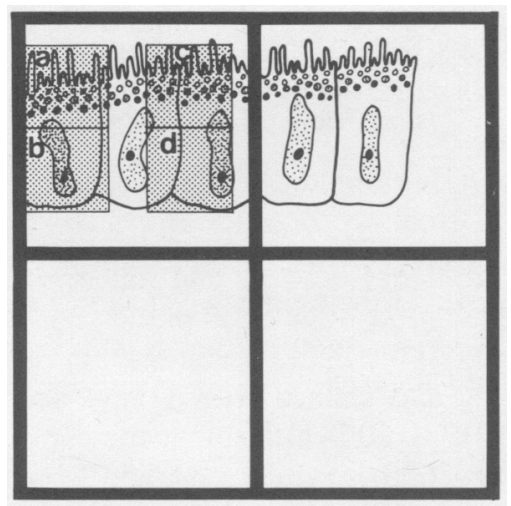

Text-fig. 2. Diagram showing the procedure of random sampling of the visceral yolk sac. The first photomicrograph is produced by positioning a random portion of the ultrathin section at the top left-hand corner (a) of the area of the copper grid illustrated and subsequently photographing random fields such as $b, c$ and $d$ (stippled).

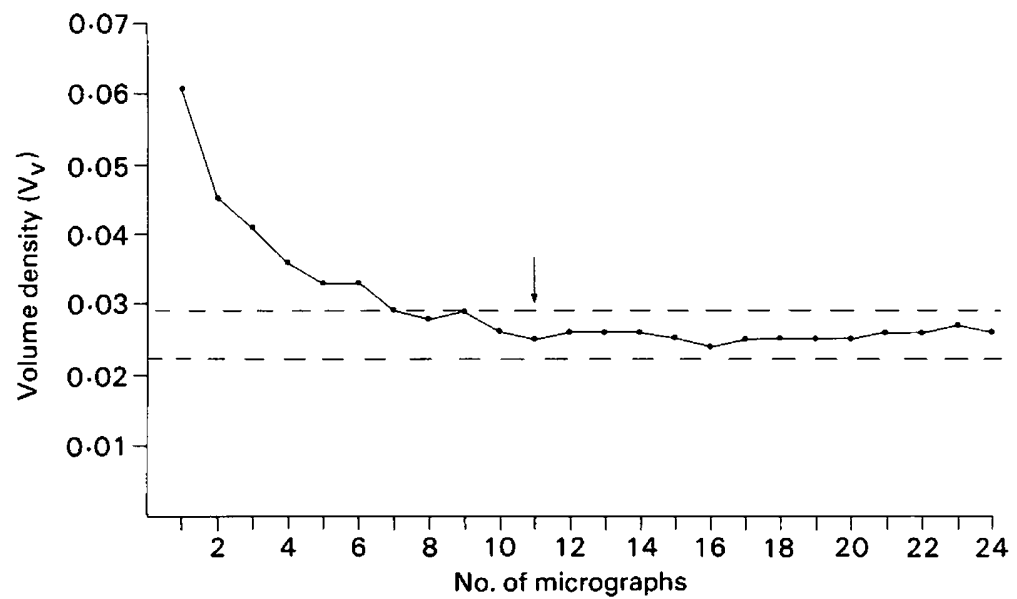

Text-fig. 3. Graph showing the determination of the minimum sample size required for morphometric analysis. This particular plot is for the volume density $\left(\mathrm{V}_{\mathrm{v}}\right)$ of type (a) endocytotic vacuoles in the rat visceral yolk sac endoderm. The minimum sample size (arrow) (i.e. the number of micrographs required to bring the $V_{v}$ estimate permanently within $10 \%$ of the final value) is 11 micrographs (see text). 
both the volume density and surface density, however, about 25-27 micrographs (i.e. well over the 11-14 minimum) were measured from each area. Data from each embryo were analysed separately. The mean values for each embryo were then combined with those from other embryos to give the overall means and standard errors quoted. Mathematical comparison of the mean results for the in-vivo and the in-vitro systems was made by using the Student's $t$ test.

\section{Results}

\section{Ultrastructure of visceral yolk sac endoderm cells}

Day 9.5 in vitro. The ultrastructural appearance of the yolk sac cells is illustrated in Pl. 1, Fig. 1. The cells were columnar and contained a moderate amount of rough endoplasmic reticulum and Golgi apparatus. The apical plasmalemma was characterized by the presence of microvili and was separated from the convoluted lateral plasmalemma by junctional complexes. The most striking feature of the cells was the population of endocytotic vacuoles which tended to occupy most of the apical cytoplasm.

Several morphological forms of the endocytotic vacuole were apparent (Pl. 1, Figs. 1 and 2): (a) small vacuoles located in the apical region of the cell at the base of the microvilli; (b) larger vacuoles varying from completely empty forms to those containing lightly flocculent material. These are situated somewhat deeper in the cytoplasm than are the small vacuoles; (c) vacuoles containing flocculent material and a homogeneous electron-dense area; and (d) vacuoles consisting entirely of electron-dense material.

By culturing rat embryos and the visceral yolk sac in the presence of electron-opaque markers (iron dextran or ferritin), it was shown that all these vacuoles are involved in endocytosis (Gupta, Gulamhusein \& Beck, 1979).

Day 9.5 in vivo. The visceral yolk sac endodermal cells in vivo looked almost identical to those in vitro except that the completely empty (type b) endocytotic vacuoles were observed only rarely compared to the in-vitro series.

Day 11.5 in vitro and in vivo. The cells were a little less tall than at 9.5 days but were otherwise similar morphologically (Pl. 2, Figs 4-6). A clearly discernible Golgi apparatus was present together with moderate amounts of rough endoplasmic and some smooth reticulum. The endocytotic vacuoles were again the most striking feature located in the apical region of the cytoplasm. The four types seen in 9.5-day yolk sac were again distinguishable and each form took up marker macromolecules.

\section{Ultrastructure of embryonic endoderm}

At 9.5 days, the visceral layer of the yolk sac is continuous with the embryonic endoderm (see Text-fig. 1) and the latter is widely exposed to the culture medium. It was therefore of interest to see if the embryonic endodermal cells take part in endocytosis. By Day 11, the embryo becomes dorsally convex and invaginated into its yolk sac so that the embryonic endoderm is no longer a surface structure. Morphometric analyses were, therefore, carried out on the embryonic endoderm cells only at 9.5 days. The ultrastructural appearance is illustrated in Pl. 1, Fig. 3. The cells were squamous with very few microvilli. There were hardly any endocytotic vacuoles although a fair amount of rough endoplasmic reticulum and Golgi apparatus could be seen.

\section{Morphometric results}

The morphometric measurements for the visceral yolk sac cells and the embryonic endodermal cells are presented in Table 1.

There were no statistically significant differences between yolk sac or embryonic endoderm 
Table 1. Results of morphometric analysis of the rat embryo endoderm

\begin{tabular}{|c|c|c|c|c|}
\hline \multirow{2}{*}{$\begin{array}{l}\text { Area of } \\
\text { the egg } \\
\text { cylinder }\end{array}$} & \multicolumn{2}{|c|}{ Day 9.5} & \multicolumn{2}{|c|}{ Day 11.5} \\
\hline & In vivo & In vitro & In vivo & In vitro \\
\hline \multicolumn{5}{|c|}{ Visceral yolk sac endoderm } \\
\hline Volume density & $15.82 \pm 0.51$ & $16.61 \pm 0.59$ & $10.68 \pm 0.21$ & $11.20 \pm 0.64$ \\
\hline Surface density & $0.58 \pm 0.02$ & $0.58 \pm 0.02$ & $0.49 \pm 0.05$ & $0.51 \pm 0.02$ \\
\hline \multicolumn{5}{|c|}{ Embryonic endoderm } \\
\hline Volume density & $2 \cdot 26 \pm 0.12$ & $2 \cdot 20 \pm 0 \cdot 18$ & - & - \\
\hline Surface density & $0.21 \pm 0.01$ & $0.18 \pm 0.02$ & - & - \\
\hline
\end{tabular}

Each value is the mean \pm s.e.m. for 6 embryos.

fixed in vivo and those prepared and grown in vitro. However, volume density and surface density values were significantly different $(P<0.001)$ for visceral endodermal cells at Days 9.5 and 11.5 and for visceral yolk sac endoderm and embryonic endoderm at Day 9.5. The values for the embryonic endoderm were much lower than any specimen of the visceral yolk sac endoderm.

\section{Discussion}

As experienced by many others (Fantel, Greenway, Juchau \& Shepard, 1979; Freeman, Beck \& Lloyd, 1981) using the technique of New (1978) whole mammalian embryo culture allows the embryo to grow as quickly in culture as in the uterus. However, one must assess whether the various 'placental' processes, such as the breakdown of histiotroph, the level of oxygenation and the methods of energy production, function in a similar manner in vivo and in culture before one can extrapolate from the in-vitro to the in-vivo situation. Based on the morphometry of the vacuolar system the present study provides evidence that the rate of endocytosis and digestion of macromolecules in the visceral yolk sac endodermal cells is similar in vitro and in vivo at 9.5 and 11.5 days although the extent of the vacuolar system at 11.5 days is significantly less than at 9.5 days. The important point is that measurement of the vacuolar system shows it to be similar in vivo and in vitro both at 9.5 and at 11.5 days. The lower measurements demonstrated on Day 11.5 could be due to the fact that the chorio-allantoic placenta would be just beginning to form in vivo.

\section{PLATE 1}

Fig. 1. Electron micrograph of the rat visceral yolk sac endoderm at 9.5 days in vitro. A large variety of endocytotic vacuoles (en.v) can be seen. $\times 2520$.

Fig. 2. A higher magnification of the visceral yolk sac endoderm at 9.5 days in vitro, showing the supranuclear region. Note the presence of different types of vacuoles. Types of vacuoles (see text) are designated as a, b, c and d. $\times 12600$.

Fig. 3. Electron micrograph of the embryonic endoderm at 9.5 days in vitro. Note the presence of a few microvilli and endocytotic vacuoles. $\times 12600$.

\section{PLATE 2}

Fig. 4. Electron micrograph of the visceral yolk sac endoderm at 11.5 days in vitro. $\times 2520$.

Fig. 5. Electron micrograph of the visceral yolk sac endoderm at 11.5 days in vitro. $\times 12600$.

Fig. 6. A higher magnification of the visceral yolk sac endoderm at 11.5 days in vivo. $\times 12600$. 
PLATE
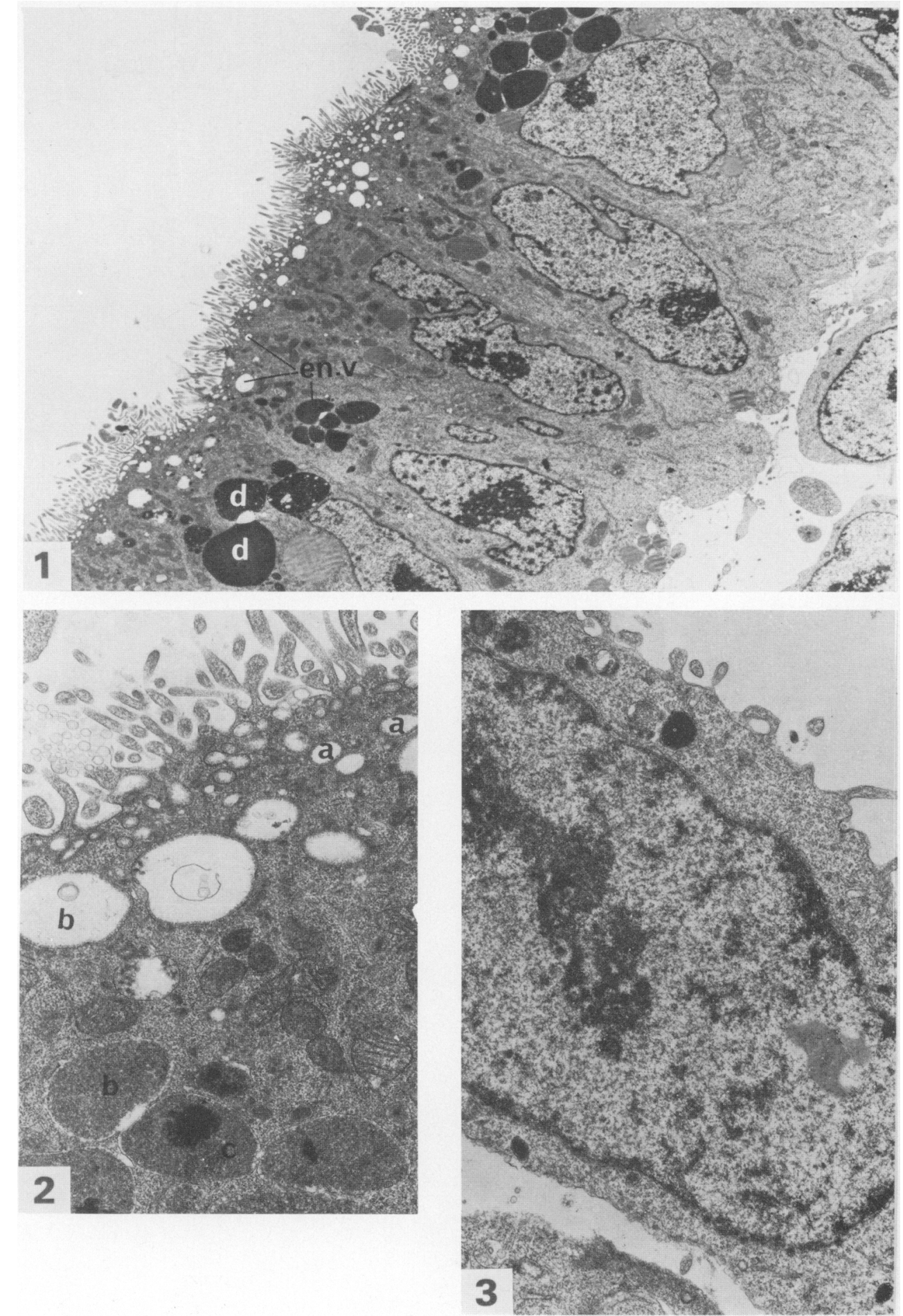

(Facing p. 244) 
PLATE 2
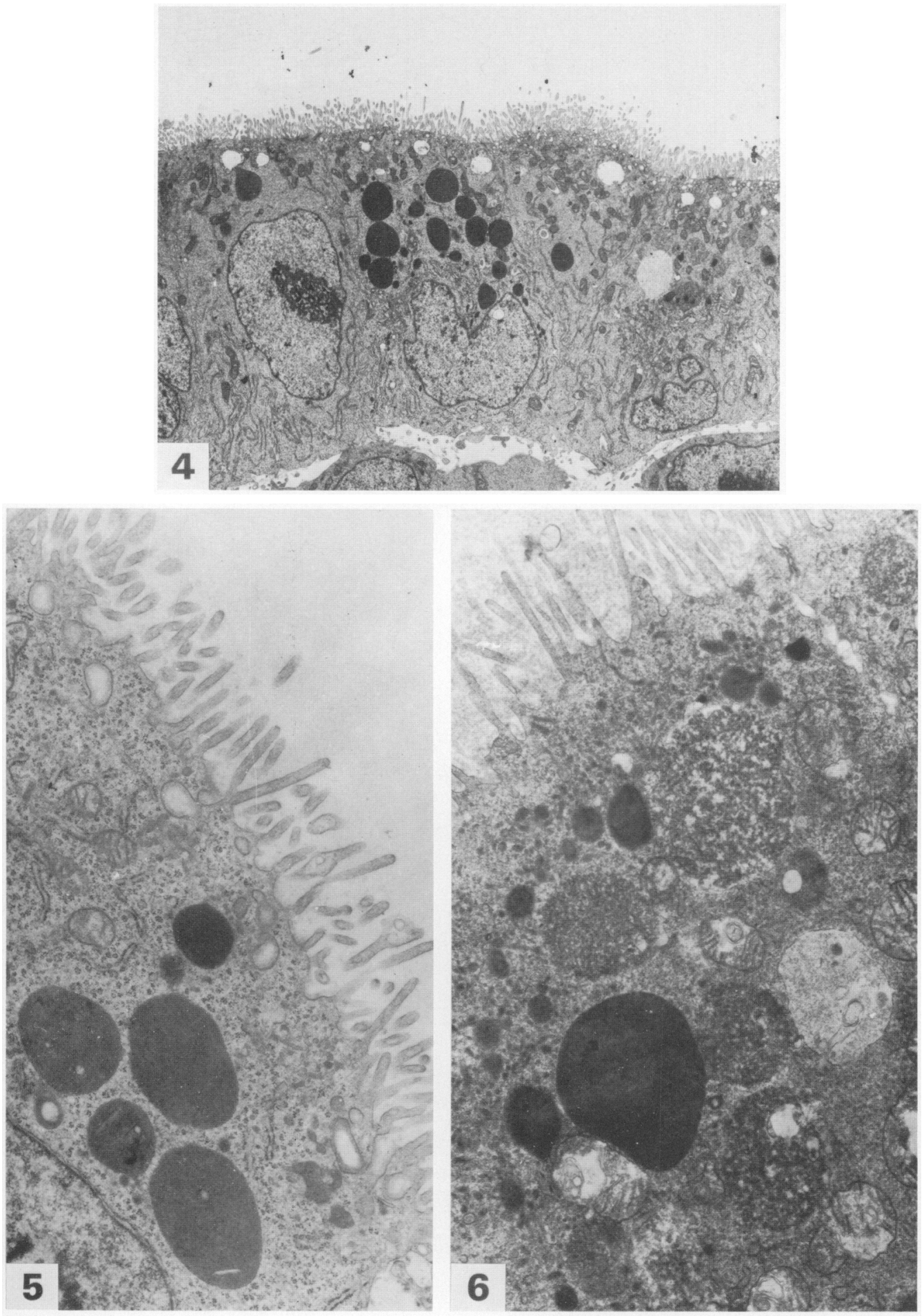
The significantly lower volume density and surface density values of the vacuoles present in the embryonic endoderm when compared to the visceral yolk sac endoderm at 9.5 days shows that the embryonic endoderm cells probably do not play a significant part in the uptake and breakdown of macromolecules, and by implication in embryonic nutrition.

The suggestion that endocytotic function is identical in culture and in vivo during the yolk sac phase of embryonic nutrition leads to more confidence in applying the technique of New (1978) to other problems in development. The system could be used to test certain teratogens to show whether they are having a direct effect on the conceptus or require the presence of the maternal organism to activate the teratogenic agent (as in cyclophosphamide; see Fantel et al., 1979).

We thank the Medical Research Council for a grant; Mrs S. Bulman and $\mathrm{Mr} \mathrm{C}$. Rigel-D'Lacey for technical assistance to APG: Dr T. M. Mayhew, Department of Anatomy, Aberdeen University, for help and advice on morphometric techniques; and Mrs A. Dean for typing the manuscript.

\section{References}

Amoroso, E.C. (1952) Placentation. In Marshall's Physiology of Reproduction, 3rd edn, Vol. II, pp. 127-311. Ed. A. S. Parkes. Longman Green, London.

Beck, F., Lloyd, J.B. \& Griffiths, A. (1967) A histochemical and biochemical study of some aspects of placental function in the rat using maternal injection of horseradish peroxidase. J. Anat. 103, 461-478.

Cockcroft, D.L. (1976) Comparison of in vitro and in vivo development of rat foetuses. Devl Biol. 48, $163-172$.

Fantel, A.G., Greenway, J.C., Juchau, M.R. \& Shepard, T.H. (1979) Teratogenic bioactivation of cyclophosphamide in vitro. Life Sciences 25, 67-72.
Freeman, S.J., Beck, F. \& Lloyd, J.B. (1981) The role of the visceral yolk sac in mediating protein utilisation by rat embryos cultured in vitro. J. Embryol. exp. Morph. 66, 223-234.

Gupta, M., Gulamhusein, A.P. \& Beck, F. (1979) Pinocytosis in the endodermal cells of the rat visceral yolk sac. J. Anat. 129, 860, Abstr.

New, D.A.T. (1978) Whole embryo culture and the study of mammalian embryos during organogenesis. Biol. Rev. 53, 81-122.

Weibel, E.R. (1969) Stereological principles for morphometry in electron microscopic cytology. Int. Rev. Cytol. 26, 235-302.

Williams, M.A. (1977) Quantitative Methods in Biology. North Holland, Amsterdam.

Received 22 August 1981 